\title{
Synthesis and Characterization of Silver-Modified Micro- Diamond as an Electrocatalyst for Oxygen Evolution and Reduction Reactions in Alkaline Medium
}

\author{
Hao Huang ${ }^{1}$, Xilong $\mathrm{Ma}^{1,2}$, Yuanyuan Liu ${ }^{1}$, Jie Hu ${ }^{1, *}$, Zhefeng $\mathrm{Xu}^{2}$, Kazuhiro Matsugi ${ }^{2}$ \\ ${ }^{1}$ State Key Laboratory of Metastable Materials Science \& Technology, Yanshan University, \\ Qinhuangdao, 066004, P.R. China \\ ${ }^{2}$ Department of Mechanical Materials Engineering, Graduate School of Engineering, Hiroshima \\ University, Higashi-Hiroshima, 739-8527, Japan \\ *E-mail: hujie@ysu.edu.cn
}

doi: $10.20964 / 2017.12 .31$

Received: 10 September 2017 / Accepted: 25 October 2017 / Published: 12 November 2017

\begin{abstract}
Silver-modified micro-diamond composites are synthesized through the chemical reduction of silver nitrate and characterized via X-ray diffraction, scanning electron microscopy (SEM), transmission electron microscopy (TEM), X-ray photoelectron spectroscopy, and Raman spectroscopy. SEM and TEM images show that silver nanoparticles $10-30 \mathrm{~nm}$ in size are uniformly deposited on the surface of the micro-diamond. Electrochemical properties are investigated through cyclic voltammetry, linear sweep voltammetry, and electrochemical impedance spectroscopy. Results show that $10 \mathrm{wt} \% \mathrm{Ag} / \mathrm{Dia}$ (silver content is $10 \%$ of the diamond) exhibits better bifunctional performance than the commercial $\mathrm{Pt} / \mathrm{C}$ (20 wt $\% \mathrm{Pt}$ on carbon) electrocatalyst under the same testing conditions. Compared with pristine micro-diamond and nanodiamond, $10 \mathrm{wt} \% \mathrm{Ag} / \mathrm{Dia}$ demonstrates lower onset potential and higher current density during oxygen reduction reaction and oxygen evolution reaction. The electron transfer number of $10 \mathrm{wt} \% \mathrm{Ag} / \mathrm{Dia}$ is approximately 3.987 at $-0.08 \mathrm{~V}$, which indicates that the reaction is almost dominated by an efficient $4 \mathrm{e}^{-}$process. The satisfactory performances of the composites provide a novel potential application for electrochemistry.
\end{abstract}

Keywords: silver; micro diamond; oxygen reduction reaction; oxygen evolution reaction; electrocatalyst.

\section{$\underline{\text { FULL TEXT }}$}

(C) 2017 The Authors. Published by ESG (www.electrochemsci.org). This article is an open access article distributed under the terms and conditions of the Creative Commons Attribution license (http://creativecommons.org/licenses/by/4.0/). 